\title{
FOCUS AND DIVERSITY IN INFORMATION SYSTEMS RESEARCH: MEETING THE DUAL DEMANDS OF A HEALTHY APPLIED DISCIPLINE
}

By: Hazel Taylor

Information School

University of Washington

Box 352840

Seattle, WA 98195-2840

U.S.A.

hztaylor@u.washington.edu

Stuart Dillon

University of Waikato

Private Bag 3105

Hamilton, 3240

NEW ZEALAND

stuart@waikato.ac.nz

\author{
Melinda Van Wingen \\ Everett Public Library \\ 2702 Hoyt Avenue \\ Everett, WA 98201 \\ U.S.A. \\ mvanwingen@ci.everett.wa.us
}

\section{Appendix A}

\section{The Author Set, Co-Citation Counts, and Bibliographies}

The starting point of any author co-citation analysis (ACA) is the identification of a set of core knowledge producers within the discipline. Clearly, if patterns of citations of key authors are used to map the development and changes in a field, then the choice of those key authors sets the boundaries and controls the patterns that will be reported. In such a broad and multidisciplinary field as IS, it is important to select authors that are recognized as the key or seminal authors in the field, and to ensure that the author set is fully representational of the breadth of the field over the span of years being investigated. Various ways of selecting authors have been used in the past, including reputation-based surveys of experts or scholars in the field (Bayer et al. 1990; Culnan 1987; Culnan et al. 1990); lists of award winners (Bayer et al. 1990); scholars named in review books or articles with an overview of the development of the field (Ellis et al. 1999); lists of highly cited authors or articles (Culnan 1986; Culnan et al. 1990; Ellis et al. 1999; Lowry et al. 2007; Ponzi 2002; White and McCain. 1998); organizational memberships (Culnan et al. 1990; McCain 1990); publication counts (Chua et al. 2002); and personal judgment (Ponzi 2002).

We used two of these approaches, publication count and reputation, to identify highly influential researchers in the IS field. While the reputational approach captures contributors who have a long tradition of influence in the field, the publication count approach is more effective 
in identifying those researchers whose influence is more recent or still emerging. Publication counts identify researchers whose papers have been recognized by leading editors and reviewers of the field as having something new and important to contribute to the IS domain. Thus, we began by selecting the most frequently published authors across five of the six journals in the AIS senior scholars' basket of journals: MIS Quarterly (MISQ), Information Systems Research (ISR), Journal of Management Information Systems (JMIS), Information Systems Journal (ISJ) and European Journal of Information Systems (EJIS) (see http://home.aisnet.org/). (The sixth journal in the AIS scholars' basket is Journal of the Association for Information Systems, which we did not include because of its relative youth, being first published in 2000). Authors of all articles published in MISQ from 1984 through 2005, all articles in JMIS, ISR, EJIS, and ISJ from their inceptions in 1984, 1990, 1991, and 1991 respectively, were counted, using the ISI Web of Science databases supplemented by manual counts for those volumes not indexed in the Web of Science databases. We used an arbitrary cut-off point of at least eight publications (excluding editorials) in MISQ, JMIS, and ISR to select the 62 most highly published researchers in these top three IS journals. An examination of contributors to EJIS and ISJ revealed a different constituency of researchers and we added a further nine authors, who each had at least four publications across these two journals.

The reputational approach enabled us to identify early contributors whose major influential publications may have been in non-IS journals, and those who have influenced the field through books rather than through journal publications. Culnan's (1987) list of authors, which she selected as being the most influential early researchers in MIS, was used as a starting point, and we added any authors on this list not already identified through the publication count. We further supplemented the author list from two sources. First, to ensure the inclusion of key foundational authors whose influential works may have been published in books or management-based journals before IS-specific journals were established, we added those researchers who have been recognized by the Association of Information Systems (AIS) for their outstanding contributions to the field. When we developed the author list in 2006, the AIS had conferred 13 LEO awards for "lifetime exceptional achievement in information systems" and 36 AIS Fellow awards, which recognize "individuals who have made outstanding contributions to the Information Systems discipline in terms of research, teaching, and service." The Leo award winners and AIS Fellows not already on the author list were added. Second, in order to expand UK/European representation, we included 10 IS authors identified on a reputational basis in a UK-based comparative study of Information Science and Information Systems research (Ellis et al. 1999).

We used cited reference searches to retrieve sets of citation records, for the years 1986-2005, to each author's works using the ISI Web of Science databases. These records formed the input for the co-citation extraction. We restricted the cited reference search to the Social Sciences database in order to reduce the number of problems arising from retrieving works by authors with the same name writing in different fields. ${ }^{1}$ Where we were aware that an author had published with one or two initials (e.g. Orlikowski W and Orlikowski WJ) or that an author's name was frequently misspelled in citations (e.g. Hirschheim is often cited as Hirscheim) we searched using both alternatives. We limited the citing references to articles in English, thus eliminating references in working papers, theses, and other works of limited accessibility to other writers in the field. Finally, we restricted the resulting sets of citing references to a broad set of information systems subject categories both to identify the most influential authors specifically in the IS field and to further reduce the likelihood of contamination from authors with the same name working in other social science fields. Five authors (Ritu Agarwal, Alok Gupta, Michael Jackson, John Ward, and Ron Weber) required intensive manual analysis to distinguish their citations from those to other authors of the same name and initial working in the IS field.

We retrieved a total of 30,059 citations referencing authors in the initial author set, comprising 7,798 unique articles. ${ }^{2}$ The initial author list comprised 117 authors as shown in Table A1, with the author set arranged in descending order of total number of IS-related citations across the twenty year period. Total citations for the 20-year period and for each five-year sub-period (1986-1990, 1991-1995, 1996-2000, 2001-2005) were recorded for each author and are shown in Table A1.

\section{Co-Citation Counts and Bibliographies}

The top 100 cited authors, with at least 85 citations each, were selected for the final author list. The next step in ACA is the extraction of cocitation counts to the author set for each time period. A database program was developed to extract co-citation counts for pairs of authors from the citation records retrieved for the final author set, for the four 5-year sub-periods from 1986 through 2005 . The co-citation counts were used

\footnotetext{
${ }^{1}$ The European Journal of Information Systems (EJIS) is officially listed as being indexed in the Science Citation Index Expanded database and not in the Social Sciences Citation Index database. However, the cited reference searches, limited to the Social Sciences database, included results from EJIS. We were advised by ISI technical support that the cited reference search uses another internal index system, in which EJIS is indexed differently. For our purposes, this different indexing worked to our advantage and enabled the problem reduction described above.

${ }^{2}$ The number of articles was less than the number of citations because many of these citations referenced more than one of the authors from the list.
} 


\begin{tabular}{|c|c|c|c|c|c|c|}
\hline Authors & Reason for Inclusion & $\begin{array}{c}\text { Total citations } \\
1986-2005\end{array}$ & $\begin{array}{l}1986- \\
1990\end{array}$ & $\begin{array}{l}1991- \\
1995\end{array}$ & $\begin{array}{l}1996- \\
2000\end{array}$ & $\begin{array}{l}2000- \\
2005\end{array}$ \\
\hline Benbasat I & MISQ, ISR, JMIS publications (39) & 751 & 72 & 136 & 242 & 301 \\
\hline Zmud RW & MISQ, ISR, JMIS publications (29) & 732 & 66 & 148 & 235 & 283 \\
\hline Ives B & MISQ, ISR, JMIS publications (34) & 714 & 96 & 206 & 236 & 176 \\
\hline Keen PGW & Culnan 1987 study & 687 & 131 & 211 & 200 & 145 \\
\hline Jarvenpaa SL & MISQ, ISR, JMIS publications (18) & 644 & 21 & 106 & 198 & 319 \\
\hline Huber GP & Culnan 1987 study & 636 & 83 & 161 & 212 & 180 \\
\hline Markus ML & MISQ, ISR, JMIS publications (11) & 614 & 51 & 112 & 215 & 236 \\
\hline Orlikowski WJ & MISQ, ISR, JMIS publications (9) & 589 & 2 & 67 & 227 & 293 \\
\hline Robey D & MISQ, ISR, JMIS publications (15) & 579 & 74 & 135 & 174 & 196 \\
\hline DeSanctis G & MISQ, ISR, JMIS publications (9) & 576 & 61 & 156 & 175 & 184 \\
\hline Rockart JF & Leo Award & 554 & 96 & 194 & 168 & 96 \\
\hline Grover V & MISQ, ISR, JMIS publications (24) & 521 & 1 & 44 & 166 & 310 \\
\hline Venkatraman $\mathrm{N}$ & Ellis et al. 1999 study & 490 & 12 & 66 & 189 & 223 \\
\hline Olson $\mathrm{MH}$ & Culnan 1987 study & 480 & 74 & 120 & 169 & 117 \\
\hline King WR & MISQ, ISR, JMIS publications (12) & 472 & 69 & 103 & 145 & 155 \\
\hline Lucas HCJ & AIS Fellow & 467 & 113 & 114 & 137 & 103 \\
\hline Dickson GW & Culnan 1987 study & 447 & 86 & 140 & 135 & 86 \\
\hline Kling $\mathrm{R}$ & Leo Award & 441 & 43 & 72 & 152 & 174 \\
\hline Nunamaker JF & MISQ, ISR, JMIS publications (36) & 435 & 18 & 120 & 160 & 137 \\
\hline McFarlan FW & Culnan 1987 study & 431 & 73 & 117 & 129 & 112 \\
\hline Wetherbe JC & MISQ, ISR, JMIS publications (8) & 428 & 45 & 120 & 165 & 98 \\
\hline Ackoff RL & Culnan 1987 study & 424 & 116 & 120 & 114 & 74 \\
\hline Todd PA & MISQ, ISR, JMIS publications (11) & 421 & 9 & 35 & 148 & 229 \\
\hline Igbaria M & MISQ, ISR, JMIS publications (14) & 420 & 3 & 62 & 154 & 201 \\
\hline Checkland P & Ellis et al. 1999 study & 395 & 54 & 113 & 114 & 114 \\
\hline Straub DW & MISQ, ISR, JMIS publications (18) & 377 & 4 & 55 & 91 & 227 \\
\hline Baroudi JJ & MISQ, ISR, JMIS publications (9) & 374 & 33 & 97 & 134 & 110 \\
\hline Lederer AL & MISQ, ISR, JMIS publications (10) & 365 & 11 & 85 & 114 & 155 \\
\hline Davis GB & MISQ, ISR, JMIS publications (11) & 362 & 69 & 100 & 101 & 92 \\
\hline Kraemer KL & MISQ, ISR, JMIS publications (12) & 352 & 33 & 83 & 97 & 139 \\
\hline Dennis AR & MISQ, ISR, JMIS publications (23) & 346 & 10 & 70 & 129 & 137 \\
\hline Hiltz SR & MISQ, ISR, JMIS publications (8) & 345 & 50 & 90 & 113 & 92 \\
\hline Alavi M & MISQ, ISR, JMIS publications (8) & 340 & 30 & 59 & 100 & 151 \\
\hline Konsynski BR & Culnan 1987 study & 331 & 33 & 109 & 98 & 91 \\
\hline Clemons EK & MISQ, ISR, JMIS publications (31) & 324 & 10 & 73 & 99 & 142 \\
\hline Hirschheim R & Ellis et al. 1999 study & 319 & 6 & 65 & 121 & 127 \\
\hline Sprague RH & MISQ, ISR, JMIS publications (9) & 311 & 90 & 110 & 77 & 34 \\
\hline Vessey I & AIS Fellow & 307 & 24 & 72 & 114 & 97 \\
\hline Swanson EB & Culnan 1987 study & 302 & 41 & 86 & 88 & 87 \\
\hline Watson RT & MISQ, ISR, JMIS publications (13) & 299 & 10 & 41 & 100 & 148 \\
\hline Vogel DR & MISQ, ISR, JMIS publications (11) & 290 & 15 & 88 & 105 & 82 \\
\hline Mumford E & Leo Award & 287 & 43 & 61 & 108 & 75 \\
\hline Valacich JS & MISQ, ISR, JMIS publications (8) & 280 & 2 & 45 & 128 & 105 \\
\hline
\end{tabular}




\begin{tabular}{|c|c|c|c|c|c|c|}
\hline Authors & Reason for Inclusion & $\begin{array}{c}\text { Total citations } \\
1986-2005\end{array}$ & $\begin{array}{l}1986- \\
1990\end{array}$ & $\begin{array}{l}1991- \\
1995\end{array}$ & $\begin{array}{l}1996- \\
2000\end{array}$ & $\begin{array}{l}2000- \\
2005\end{array}$ \\
\hline Ginzberg MJ & AIS Fellow & 279 & 51 & 78 & 79 & 71 \\
\hline Whinston $A B$ & MISQ, ISR, JMIS publications (17) & 269 & 13 & 32 & 88 & 136 \\
\hline King JL & MISQ, ISR, JMIS publications (25) & 268 & 35 & 88 & 80 & 65 \\
\hline Willcocks LP & ISJ/EJIS publications (9) & 268 & $\mathrm{n} / \mathrm{a}$ & 16 & 112 & 140 \\
\hline Mason RO & Leo Award & 267 & 63 & 77 & 75 & 52 \\
\hline Higgins CA & MISQ, ISR, JMIS publications (9) & 265 & 4 & 38 & 88 & 135 \\
\hline Barki H & MISQ, ISR, JMIS publications (11) & 257 & 4 & 44 & 81 & 128 \\
\hline Churchman CW & Leo Award & 257 & 76 & 76 & 56 & 49 \\
\hline Goodhue DL & MISQ, ISR, JMIS publications (8) & 249 & 1 & 31 & 83 & 134 \\
\hline Walsham G & ISJ/EJIS publications (5) & 248 & 5 & 26 & 95 & 122 \\
\hline Nolan RL & Culnan 1987 study & 246 & 57 & 71 & 75 & 43 \\
\hline Tam KY & MISQ, ISR, JMIS publications (13) & 242 & 1 & 27 & 83 & 131 \\
\hline Bostrom RP & MISQ, ISR, JMIS publications (9) & 240 & 13 & 60 & 91 & 76 \\
\hline Guimaraes T & MISQ, ISR, JMIS publications (9) & 236 & 23 & 46 & 97 & 70 \\
\hline Lyytinen K & AIS Fellow & 231 & 6 & 37 & 76 & 112 \\
\hline Eindor $\mathrm{P}$ & AIS Fellow & 223 & 35 & 56 & 78 & 54 \\
\hline Galliers RD & Ellis et al. 1999 study & 220 & 1 & 35 & 101 & 83 \\
\hline Mukhopadhyay T & MISQ, ISR, JMIS publications (14) & 217 & $\mathrm{n} / \mathrm{a}$ & 10 & 79 & 128 \\
\hline Agarwal R & MISQ, ISR, JMIS publications (15) & 216 & $\mathrm{n} / \mathrm{a}$ & 12 & 47 & 157 \\
\hline Lee AS & MISQ, ISR, JMIS publications (20) & 203 & 1 & 24 & 92 & 86 \\
\hline Watson $\mathrm{HJ}$ & MISQ, ISR, JMIS publications (11) & 196 & 28 & 49 & 63 & 56 \\
\hline McKenney JL & AIS Fellow & 194 & 35 & 48 & 66 & 45 \\
\hline Mingers $\mathrm{J}$ & Ellis et al. 1999 study & 190 & 9 & 50 & 57 & 74 \\
\hline Gorry GA & Culnan 1987 study & 188 & 53 & 65 & 47 & 23 \\
\hline Chen PPS & Culnan 1987 study & 184 & 46 & 64 & 39 & 35 \\
\hline Barua A & MISQ, ISR, JMIS publications (9) & 182 & $\mathrm{n} / \mathrm{a}$ & 4 & 71 & 107 \\
\hline Sambamurthy V & MISQ, ISR, JMIS publications (15) & 178 & $\mathrm{n} / \mathrm{a}$ & 12 & 41 & 125 \\
\hline Leidner DE & MISQ, ISR, JMIS publications (9) & 171 & $\mathrm{n} / \mathrm{a}$ & 3 & 31 & 137 \\
\hline Chin WW & MISQ, ISR, JMIS publications (9) & 170 & $\mathrm{n} / \mathrm{a}$ & 2 & 33 & 135 \\
\hline Boland RJ & Culnan 1987 study & 168 & 16 & 31 & 51 & 70 \\
\hline Lacity MC & ISJ/EJIS publications (4) & 168 & $\mathrm{n} / \mathrm{a}$ & 16 & 67 & 85 \\
\hline Couger JD & Leo Award & 166 & 23 & 54 & 54 & 35 \\
\hline Rivard S & MISQ, ISR, JMIS publications (8) & 163 & 17 & 46 & 43 & 57 \\
\hline Kettinger WJ & MISQ, ISR, JMIS publications (10) & 160 & 1 & 3 & 55 & 101 \\
\hline Kauffman RJ & MISQ, ISR, JMIS publications (20) & 153 & $\mathrm{n} / \mathrm{a}$ & 14 & 35 & 104 \\
\hline Kriebel $\mathrm{CH}$ & Culnan 1987 study & 152 & 13 & 18 & 56 & 65 \\
\hline Anthony RN & Culnan 1987 study & 150 & 41 & 46 & 35 & 28 \\
\hline Liang TP & AIS Fellow & 147 & 4 & 45 & 46 & 52 \\
\hline Chervany NL & Culnan 1987 study & 144 & 19 & 16 & 18 & 91 \\
\hline Wiseman CM & Ellis et al. 1999 study & 139 & 21 & 52 & 42 & 24 \\
\hline Saunders CS & MISQ, ISR, JMIS publications (8) & 134 & 5 & 12 & 37 & 80 \\
\hline Mclean ER & AIS Fellow & 132 & 25 & 34 & 31 & 42 \\
\hline Weber R & MISQ, ISR, JMIS publications (17) & 127 & 13 & 19 & 34 & 61 \\
\hline
\end{tabular}




\begin{tabular}{|c|c|c|c|c|c|c|}
\hline Authors & Reason for Inclusion & $\begin{array}{c}\text { Total citations } \\
1986-2005\end{array}$ & $\begin{array}{l}1986- \\
1990\end{array}$ & $\begin{array}{l}1991- \\
1995\end{array}$ & $\begin{array}{l}1996- \\
2000\end{array}$ & $\begin{array}{l}2000- \\
2005\end{array}$ \\
\hline Gray P & Leo Award & 125 & 30 & 29 & 34 & 32 \\
\hline Alter S & Culnan 1987 study & 121 & 18 & 27 & 32 & 44 \\
\hline Land FF & Leo Award & 119 & 15 & 25 & 51 & 28 \\
\hline Bjornandersen $\mathrm{N}$ & AIS Fellow & 118 & 20 & 37 & 32 & 29 \\
\hline Zwass V & MISQ, ISR, JMIS publications (33) & 106 & 1 & 3 & 33 & 69 \\
\hline Vitale MR & AIS Fellow & 105 & 4 & 32 & 36 & 33 \\
\hline Baskerville RL & ISJ/EJIS publications (8) & 103 & $\mathrm{n} / \mathrm{a}$ & 5 & 34 & 64 \\
\hline Wei KK & MISQ, ISR, JMIS publications (9) & 103 & $\mathrm{n} / \mathrm{a}$ & 2 & 36 & 65 \\
\hline Munro MC & Culnan 1987 study & 101 & 23 & 36 & 20 & 22 \\
\hline Cavaye ALM & ISJ/EJIS publications (4) & 99 & $\mathrm{n} / \mathrm{a}$ & 0 & 32 & 67 \\
\hline Ciborra C & Ellis et al. 1999 study & 93 & 5 & 9 & 36 & 43 \\
\hline Ward J & Ellis et al. 1999 study & 88 & $\mathrm{n} / \mathrm{a}$ & 8 & 30 & 50 \\
\hline Jenkins AM & Culnan 1987 study & 86 & 12 & 25 & 34 & 15 \\
\hline Galletta DF & AIS Fellow & 85 & 2 & 25 & 40 & 18 \\
\hline Smithson S & ISJ/EJIS publications (6) & 72 & $\mathrm{n} / \mathrm{a}$ & 4 & 31 & 37 \\
\hline O'Keefe, RM & ISJ/EJIS publications (4) & 68 & 2 & 15 & 21 & 30 \\
\hline Jackson MA & Ellis et al. 1999 study & 66 & 16 & 21 & 19 & 10 \\
\hline Neumann S & AIS Fellow & 63 & 6 & 12 & 30 & 15 \\
\hline Karimi J & MISQ, ISR, JMIS publications (8) & 62 & 3 & 20 & 13 & 26 \\
\hline Mathiassen L & ISJ/EJIS publications (4) & 59 & 4 & 5 & 19 & 31 \\
\hline Briggs $R O$ & MISQ, ISR, JMIS publications (17) & 45 & $\mathrm{n} / \mathrm{a}$ & 3 & 9 & 33 \\
\hline Kozar KA & MISQ, ISR, JMIS publications (8) & 39 & 4 & 6 & 16 & 13 \\
\hline Avergou C & AIS Fellow & 37 & $\mathrm{n} / \mathrm{a}$ & 4 & 8 & 25 \\
\hline Gupta A & MISQ, ISR, JMIS publications (8) & 34 & $\mathrm{n} / \mathrm{a}$ & 0 & 6 & 28 \\
\hline Carlson ED & Culnan 1987 study & 28 & 13 & 9 & 5 & 1 \\
\hline Mookerjee VS & MISQ, ISR, JMIS publications (8) & 25 & $\mathrm{n} / \mathrm{a}$ & 2 & 6 & 17 \\
\hline Weber BW & MISQ, ISR, JMIS publications (14) & 24 & $\mathrm{n} / \mathrm{a}$ & 1 & 7 & 16 \\
\hline Langefors $B$ & Leo Award & 20 & 8 & 4 & 6 & 2 \\
\hline De Vreede GJ & MISQ, ISR, JMIS publications (9) & 18 & $\mathrm{n} / \mathrm{a}$ & 0 & 8 & 10 \\
\hline Stowell, $F$ & ISJ/EJIS publications (5) & 17 & $\mathrm{n} / \mathrm{a}$ & 0 & 10 & 7 \\
\hline Choudhary V & MISQ, ISR, JMIS publications (8) & 14 & $\mathrm{n} / \mathrm{a}$ & 0 & 2 & 12 \\
\hline \multicolumn{2}{|c|}{ TOTAL CITATIONS } & 30,059 & 2,927 & 6,323 & 9,680 & 11,129 \\
\hline
\end{tabular}

*We selected authors sequentially, using the following five criteria in order: (1) at least eight publications across MISQ, ISR, and JMIS; (2) at least four publications in ISJ and EJIS; (3) included in Culnan's (1987) study; (4) LEO award or AIS Fellow; (5) included in Ellis et al.'s (1999) study. While some authors met multiple criteria for inclusion, the reason for inclusion column shows only the first criterion met by each author.

MISQ = MIS Quarterly; ISR = Information Systems Research; JMIS = Journal of Management Information Systems; ISJ = Information Systems Journal; EJIS = European Journal of Information Systems.

$\mathrm{n} / \mathrm{a}$ indicates no publications to cite in this period.

Italicized authors were dropped from the final set. 
as input for the factor and MDS analyses, the two analytic techniques used most prevalently in co-citation research (McCain 1990; White and McCain 1998). The database program also extracted co-citation bibliographies for the groups of foundational authors that loaded on each factor, as described in Appendix B. The bibliographies contained all articles that co-cited any pair of the authors in the factor. A total of 3,818 unique articles citing at least two of the top 100 authors within a factor were retrieved across all factors and periods. The articles obtained were coded for research theme, as described in Appendix C.

\section{Appendix B}

\section{Factor Analyses}

In co-citation research, factor analysis is performed on a matrix of co-citation counts, in which the same list of foundational authors comprises both the column and row headings, and each cell value represents the number of articles that have cited the relevant pair of foundational authors. In co-citation matrices, no meaningful counts can be applied to cells along the diagonal (i.e., the number of times an author is co-cited with $\mathrm{him} /$ herself), although factor analyses requires numbers in these cells. Hence we used mean substitution, the most commonly applied method for addressing this problem in co-citation research (White and McCain 1998). Following the convention typically used in co-citation research (McCain 1990), authors with a mean of less than four co-citations within a period were dropped from that period in order to ensure interpretable results. In order to drop as few authors as possible, an iterative process was used in which the author with the lowest mean co-citation was removed from the data matrix until all remaining authors met the threshold, resulting in the retention of 37 authors for the 1986-1990 period; 63 for the 1991-1995 period; 72 for the 1996-2000 period; and 79 for the 2001-2005 period.

The raw co-citation matrices for each period were used as input for principal component factor analyses with varimax rotation, using SPSS. The results of the factor analyses for each of the four 5-year periods are shown in Tables B1 through B4. As is typical in this type of factor analysis, the factors are not mutually exclusive (i.e., the authors do not load exclusively on a single factor), so interpretation of factors is based on those authors with high loadings (McCain 1990). Authors with high loadings on two or more factors are considered to be contributing to two or more subfields within the discipline (White and McCain 1998). Following typical ACA conventions (Culnan 1987; McCain 1990; White and McCain 1998), authors loading with absolute values less than 0.4 on any factor were suppressed. (For the 1996-2000 period the absolute threshold loading was set at 0.38 in order to ensure that all authors in this set loaded on at least one factor.) The number of factors identified for each period was determined primarily from examinations of scree plots and secondarily from a consideration of the number of factors with eigenvalues greater than one. Results yielded a four-factor solution for the first (1986-1990) period, accounting for 74.8 percent of the variance; five-factor solutions for each of the next two periods (1991-1995 and 1996-2000) accounting for 78.7 percent and 73.8 percent of the variance respectively; and a six-factor solution for the final (2001-2005) period, accounting for 76.4 percent of the variance. Our procedures for interpreting the factors and assigning the factor names shown in Tables B1 through B4 are discussed in Appendix C. 


\begin{tabular}{|c|c|c|c|c|}
\hline $\begin{array}{c}1986-1990 \text { Authors } \\
\text { ( } 24 \text { mean co-citations) }\end{array}$ & $\begin{array}{l}\text { IS Development \& } \\
\text { Use Thematic } \\
\text { Miscellany }\end{array}$ & IS Strategy & $\begin{array}{c}\text { Group Work \& } \\
\text { Decision Support }\end{array}$ & $\begin{array}{c}\text { Foundations \& Reference } \\
\text { Disciplines }\end{array}$ \\
\hline Swanson & 0.901 & & & \\
\hline Mumford & 0.898 & & & \\
\hline Markus & 0.887 & & & \\
\hline Robey & 0.875 & & & \\
\hline Kling & 0.873 & & & \\
\hline Ginzberg & 0.859 & & & \\
\hline Baroudi & 0.832 & & & \\
\hline Olson & 0.812 & & & \\
\hline Lucas & 0.792 & & & \\
\hline Ives & 0.742 & 0.418 & & \\
\hline Zmud & 0.651 & 0.576 & & \\
\hline Dickson & 0.608 & & 0.412 & \\
\hline Keen & 0.537 & 0.424 & & \\
\hline McLean & & 0.917 & & \\
\hline McKenney & & 0.903 & & \\
\hline Nolan & & 0.873 & & \\
\hline McFarlan & & 0.831 & & \\
\hline Wetherbe & & 0.799 & & \\
\hline Rockart & 0.414 & 0.774 & & \\
\hline KingWR & 0.544 & 0.716 & & \\
\hline Eindor & 0.589 & 0.711 & & \\
\hline Davis & 0.585 & 0.698 & & \\
\hline Alavi & 0.480 & 0.568 & & \\
\hline Hiltz & & & 0.835 & \\
\hline DeSanctis & & & 0.770 & \\
\hline Huber & & & 0.734 & \\
\hline Konsynski & & & 0.713 & \\
\hline Benbasat & 0.435 & & 0.685 & \\
\hline Kraemer & & & 0.678 & \\
\hline Chervany & & & 0.541 & 0.423 \\
\hline KingJL & & 0.440 & 0.523 & \\
\hline Sprague & & & 0.474 & \\
\hline Mason & & & & 0.813 \\
\hline Gorry & & & & 0.770 \\
\hline Ackoff & & & & 0.740 \\
\hline Churchman & & & & 0.690 \\
\hline Anthony & & 0.562 & & 0.591 \\
\hline$\%$ Variance & 28.9 & 23.2 & 12.6 & 10.1 \\
\hline
\end{tabular}




\begin{tabular}{|c|c|c|c|c|c|}
\hline $\begin{array}{l}\text { 1991-1995 Authors } \\
\text { ( } \geq 4 \text { mean co- } \\
\text { citations) }\end{array}$ & $\begin{array}{c}\text { IS Development \& } \\
\text { Use Thematic } \\
\text { Miscellany }\end{array}$ & $\begin{array}{c}\text { Qualitative } \\
\text { Methods Thematic } \\
\text { Miscellany }\end{array}$ & $\begin{array}{c}\text { IS } \\
\text { Strategy }\end{array}$ & $\begin{array}{l}\text { Group Work \& } \\
\text { Decision } \\
\text { Support }\end{array}$ & $\begin{array}{l}\text { Foundations \& } \\
\text { Reference } \\
\text { Disciplines }\end{array}$ \\
\hline Igbaria & 0.898 & & & & \\
\hline Guimaraes & 0.857 & & & & \\
\hline Rivard & 0.847 & & & & \\
\hline Lucas & 0.799 & 0.452 & & & \\
\hline Swanson & 0.796 & 0.464 & & & \\
\hline Alavi & 0.793 & & & & \\
\hline Barki & 0.792 & 0.492 & & & \\
\hline Baroudi & 0.771 & & & & \\
\hline Eindor & 0.767 & & 0.445 & & \\
\hline Vessey & 0.765 & & & & \\
\hline Davis & 0.752 & & & & \\
\hline Ginzberg & 0.747 & 0.531 & & & \\
\hline Olson & 0.740 & 0.435 & & & \\
\hline Zmud & 0.735 & & 0.412 & & \\
\hline Ives & 0.706 & & & & \\
\hline Straub & 0.695 & & & & \\
\hline Dickson & 0.662 & & & 0.475 & \\
\hline Robey & 0.661 & 0.568 & & & \\
\hline Benbasat & 0.646 & & & 0.473 & \\
\hline Keen & 0.584 & & & & \\
\hline Couger & 0.579 & & & & \\
\hline WatsonHJ & 0.548 & & 0.453 & & 0.460 \\
\hline Bjornandersen & & 0.886 & & & \\
\hline Boland & & 0.862 & & & \\
\hline Mumford & & 0.849 & & & \\
\hline Lyytinen & & 0.823 & & & \\
\hline Orlikowski & & 0.809 & & & \\
\hline Hirschheim & & 0.780 & & & \\
\hline Kling & & 0.773 & & & \\
\hline Markus & 0.478 & 0.686 & & & \\
\hline Bostrom & 0.616 & 0.627 & & & \\
\hline Wiseman & & & 0.955 & & \\
\hline Vitale & & & 0.931 & & \\
\hline Venkatraman & & & 0.925 & & \\
\hline McKenney & & & 0.917 & & \\
\hline Clemons & & & 0.906 & & \\
\hline McFarlan & & & 0.846 & & \\
\hline Lederer & 0.509 & & 0.769 & & \\
\hline Konsynski & & & 0.763 & 0.459 & \\
\hline Nolan & 0.453 & & 0.760 & & \\
\hline Grover & & & 0.752 & & \\
\hline Goodhue & 0.424 & & 0.744 & & \\
\hline Rockart & 0.470 & & 0.711 & & \\
\hline
\end{tabular}




\begin{tabular}{|c|c|c|c|c|c|}
\hline $\begin{array}{l}\text { 1991-1995 Authors } \\
\text { ( } \geq 4 \text { mean co- } \\
\text { citations) }\end{array}$ & $\begin{array}{l}\text { IS Development \& } \\
\text { Use Thematic } \\
\text { Miscellany }\end{array}$ & $\begin{array}{c}\text { Qualitative } \\
\text { Methods Thematic } \\
\text { Miscellany }\end{array}$ & $\begin{array}{c}\text { IS } \\
\text { Strategy }\end{array}$ & $\begin{array}{l}\text { Group Work \& } \\
\text { Decision } \\
\text { Support }\end{array}$ & $\begin{array}{l}\text { Foundations \& } \\
\text { Reference } \\
\text { Disciplines }\end{array}$ \\
\hline KingWR & 0.633 & & 0.671 & & \\
\hline McLean & 0.625 & & 0.656 & & \\
\hline Wetherbe & 0.595 & & 0.606 & & \\
\hline Hiltz & & & & 0.940 & \\
\hline WatsonRT & & & & 0.906 & \\
\hline Valacich & & & & 0.887 & \\
\hline Dennis & & & & 0.866 & \\
\hline Vogel & & & & 0.856 & \\
\hline Nunamaker & & & & 0.844 & \\
\hline Kraemer & & & & 0.813 & \\
\hline DeSanctis & & & & 0.808 & \\
\hline Huber & & & & 0.794 & \\
\hline KingJL & & & & 0.665 & \\
\hline Jarvenpaa & 0.489 & & & 0.628 & \\
\hline Gorry & 0.454 & & & & 0.719 \\
\hline Ackoff & & & & & 0.707 \\
\hline Mason & & & & 0.422 & 0.695 \\
\hline Churchman & & 0.477 & & & 0.669 \\
\hline Sprague & & & & 0.412 & 0.600 \\
\hline Checkland & & 0.484 & & & 0.531 \\
\hline$\%$ Variance & 25.9 & 13.7 & 18.5 & 14.5 & 6.1 \\
\hline
\end{tabular}

\section{Table B3. 1996-2000 Author Factor Loadings (.38 or Higher)}

\begin{tabular}{|l|c|c|c|c|c|}
\hline $\begin{array}{c}\text { 1996-2000 Authors } \\
\text { ( 4 mean co- } \\
\text { citations) }\end{array}$ & $\begin{array}{c}\text { IS Development \& } \\
\text { Use Thematic } \\
\text { Miscellany }\end{array}$ & $\begin{array}{c}\text { Qualitative } \\
\text { Methods Thematic } \\
\text { Miscellany }\end{array}$ & $\begin{array}{c}\text { IS } \\
\text { Strategy }\end{array}$ & $\begin{array}{c}\text { Group Work \& } \\
\text { Decision } \\
\text { Support }\end{array}$ & $\begin{array}{c}\text { Inter-Business } \\
\text { Systems }\end{array}$ \\
\hline Barki & 0.917 & & & & \\
\hline Igbaria & 0.882 & & & & \\
\hline Rivard & 0.873 & & & & \\
\hline Guimaraes & 0.853 & & & & \\
\hline Higgins & 0.823 & & & & \\
\hline Baroudi & 0.807 & & & & \\
\hline Lucas & 0.803 & 0.383 & & & \\
\hline Ginzberg & 0.802 & & 0.427 & & \\
\hline Eindor & 0.800 & & & & \\
\hline WatsonHJ & 0.799 & & & & \\
\hline Olson & 0.792 & & & & \\
\hline Straub & 0.783 & & 0.456 & & \\
\hline Wetherbe & 0.757 & & & & \\
\hline Galletta & 0.746 & & & & \\
\hline Ives & 0.740 & & & & \\
\hline
\end{tabular}




\begin{tabular}{|c|c|c|c|c|c|}
\hline $\begin{array}{l}\text { 1996-2000 Authors } \\
\text { ( } \geq 4 \text { mean co- } \\
\text { citations) }\end{array}$ & $\begin{array}{c}\text { IS Development \& } \\
\text { Use Thematic } \\
\text { Miscellany }\end{array}$ & $\begin{array}{c}\text { Qualitative } \\
\text { Methods Thematic } \\
\text { Miscellany }\end{array}$ & $\begin{array}{c}\text { IS } \\
\text { Strategy }\end{array}$ & $\begin{array}{l}\text { Group Work \& } \\
\text { Decision } \\
\text { Support }\end{array}$ & $\begin{array}{l}\text { Inter-Business } \\
\text { Systems }\end{array}$ \\
\hline Swanson & 0.727 & 0.490 & & & \\
\hline Zmud & 0.681 & & 0.456 & & \\
\hline Goodhue & 0.678 & & 0.407 & & \\
\hline Davis & 0.674 & & 0.390 & & \\
\hline Robey & 0.658 & 0.584 & & & \\
\hline Couger & 0.637 & & & & \\
\hline Todd & 0.630 & & & 0.437 & \\
\hline Vessey & 0.610 & & & 0.460 & \\
\hline Sprague & 0.454 & & & 0.426 & \\
\hline Boland & & 0.867 & & & \\
\hline Lyytinen & & 0.849 & & & \\
\hline Lee & & 0.846 & & & \\
\hline Walsham & & 0.831 & & & \\
\hline Kling & & 0.829 & & & \\
\hline Hirschheim & & 0.803 & & & \\
\hline Checkland & & 0.767 & & & \\
\hline Mumford & 0.415 & 0.758 & & & \\
\hline Orlikowski & & 0.742 & & & \\
\hline Land & & 0.702 & 0.397 & & \\
\hline Markus & 0.403 & 0.695 & & & \\
\hline Wiseman & & & 0.895 & & \\
\hline McKenney & & & 0.856 & & \\
\hline Nolan & & & 0.848 & & \\
\hline McFarlan & & & 0.816 & & \\
\hline Venkatraman & & & 0.796 & & \\
\hline Galliers & & 0.495 & 0.725 & & \\
\hline Rockart & 0.476 & & 0.717 & & \\
\hline Clemons & & & 0.714 & & 0.533 \\
\hline KingWR & 0.570 & & 0.700 & & \\
\hline Lederer & 0.564 & & 0.695 & & \\
\hline Grover & 0.470 & & 0.639 & & \\
\hline Lacity & & 0.412 & 0.605 & & \\
\hline Kettinger & & & 0.588 & & \\
\hline Willcocks & & 0.511 & 0.585 & & \\
\hline Konsynski & & & 0.577 & & 0.498 \\
\hline Keen & 0.522 & 0.427 & 0.546 & & \\
\hline KingJL & & & 0.382 & & \\
\hline WatsonRT & & & & 0.925 & \\
\hline Huber & & & & 0.910 & \\
\hline Hiltz & & & & 0.904 & \\
\hline DeSanctis & & & & 0.891 & \\
\hline Vogel & & & & 0.877 & \\
\hline
\end{tabular}




\begin{tabular}{|c|c|c|c|c|c|}
\hline $\begin{array}{l}\text { 1996-2000 Authors } \\
\text { ( } \geq 4 \text { mean co- } \\
\text { citations) }\end{array}$ & $\begin{array}{c}\text { IS Development \& } \\
\text { Use Thematic } \\
\text { Miscellany }\end{array}$ & $\begin{array}{c}\text { Qualitative } \\
\text { Methods Thematic } \\
\text { Miscellany }\end{array}$ & $\begin{array}{c}\text { IS } \\
\text { Strategy }\end{array}$ & $\begin{array}{l}\text { Group Work \& } \\
\text { Decision } \\
\text { Support }\end{array}$ & $\begin{array}{l}\text { Inter-Business } \\
\text { Systems }\end{array}$ \\
\hline Valacich & & & & 0.852 & \\
\hline Nunamaker & & & & 0.852 & \\
\hline Dennis & & & & 0.849 & \\
\hline Dickson & & & & 0.813 & \\
\hline Jarvenpaa & & & & 0.804 & \\
\hline Sambamurthy & & & 0.423 & 0.804 & \\
\hline Kraemer & & & & 0.746 & \\
\hline Benbasat & & & & 0.685 & \\
\hline Bostrom & 0.591 & & & 0.648 & \\
\hline Alavi & 0.611 & & & 0.635 & \\
\hline Mason & & & & 0.548 & \\
\hline Kriebel & & & & & 0.794 \\
\hline Barua & & & & & 0.789 \\
\hline Mukhopadhyay & & & & & 0.784 \\
\hline Whinston & & & & 0.414 & 0.675 \\
\hline$\%$ Variance & 24.1 & 13.4 & 14.9 & 16.4 & 5.0 \\
\hline
\end{tabular}

\begin{tabular}{|c|c|c|c|c|c|c|}
\hline $\begin{array}{l}\text { 2001-2005 Authors } \\
\text { ( } \geq 4 \text { mean co- } \\
\text { citations) }\end{array}$ & $\begin{array}{c}\text { IS Development \& } \\
\text { Use Thematic } \\
\text { Miscellany }\end{array}$ & $\begin{array}{c}\text { Internet } \\
\text { Application } \\
\text { s Thematic } \\
\text { Miscellany }\end{array}$ & $\begin{array}{l}\text { Qualitative } \\
\text { Methods } \\
\text { Thematic } \\
\text { Miscellany }\end{array}$ & IS Strategy & $\begin{array}{l}\text { Group Work } \\
\text { \& Decision } \\
\text { Support }\end{array}$ & $\begin{array}{c}\text { Inter- } \\
\text { Business } \\
\text { Systems }\end{array}$ \\
\hline Ginzberg & 0.782 & & & & & \\
\hline Baroudi & 0.775 & & & & & \\
\hline Olson & 0.766 & & & & & \\
\hline Guimaraes & 0.733 & 0.420 & & & & \\
\hline Ives & 0.677 & & & & & \\
\hline Eindor & 0.635 & & & 0.618 & & \\
\hline WatsonHJ & 0.532 & 0.486 & & & & \\
\hline Agarwal & & 0.910 & & & & \\
\hline Higgins & & 0.909 & & & & \\
\hline Chin & & 0.884 & & & & \\
\hline Chervany & & 0.883 & & & & \\
\hline Igbaria & & 0.842 & & & & \\
\hline Tam & & 0.839 & & & & \\
\hline Todd & & 0.823 & & & & \\
\hline Cavaye & & 0.816 & & & & \\
\hline Straub & & 0.808 & & & & \\
\hline Lucas & 0.498 & 0.687 & & & & \\
\hline Goodhue & & 0.655 & & 0.526 & & \\
\hline
\end{tabular}




\begin{tabular}{|c|c|c|c|c|c|c|}
\hline $\begin{array}{l}\text { 2001-2005 Authors } \\
\text { ( } \geq 4 \text { mean co- } \\
\text { citations) }\end{array}$ & $\begin{array}{l}\text { IS Development \& } \\
\text { Use Thematic } \\
\text { Miscellany }\end{array}$ & $\begin{array}{c}\text { Internet } \\
\text { Application } \\
\text { s Thematic } \\
\text { Miscellany }\end{array}$ & $\begin{array}{l}\text { Qualitative } \\
\text { Methods } \\
\text { Thematic } \\
\text { Miscellany }\end{array}$ & IS Strategy & $\begin{array}{l}\text { Group Work } \\
\text { \& Decision } \\
\text { Support }\end{array}$ & $\begin{array}{c}\text { Inter- } \\
\text { Business } \\
\text { Systems }\end{array}$ \\
\hline Barki & 0.580 & 0.645 & & & & \\
\hline Davis & & 0.604 & 0.402 & & & \\
\hline Vessey & & 0.497 & & & 0.425 & \\
\hline Walsham & & & 0.914 & & & \\
\hline Baskerville & & & 0.912 & & & \\
\hline Kling & & & 0.863 & & & \\
\hline Lee & & & 0.856 & & & \\
\hline Mumford & & & 0.851 & & & \\
\hline Lyytinen & & & 0.842 & & & \\
\hline Checkland & & & 0.804 & & & \\
\hline Hirschheim & & & 0.750 & & & \\
\hline Markus & & & 0.722 & & & \\
\hline Robey & & & 0.711 & & & \\
\hline Orlikowski & & & 0.699 & & & \\
\hline WeberR & & & 0.616 & & & \\
\hline Swanson & 0.496 & & 0.579 & & & \\
\hline KingJL & & & 0.453 & & & \\
\hline Vitale & & & & 0.846 & & \\
\hline Ward & & & & 0.805 & & \\
\hline McFarlan & & & & 0.800 & & \\
\hline McKenney & & & & 0.799 & & \\
\hline Venkatraman & & & & 0.796 & & \\
\hline Rockart & & & & 0.787 & & \\
\hline Lederer & & & & 0.774 & & \\
\hline KingWR & 0.465 & & & 0.735 & & \\
\hline Galliers & & & 0.564 & 0.686 & & \\
\hline Kettinger & 0.432 & & & 0.678 & & \\
\hline Clemons & & & & 0.673 & & 0.620 \\
\hline Grover & & 0.409 & & 0.664 & & \\
\hline Keen & & & & 0.660 & & \\
\hline Sambamurthy & & 0.529 & & 0.630 & & \\
\hline Wetherbe & 0.530 & 0.418 & & 0.606 & & \\
\hline McLean & & & & 0.597 & & \\
\hline Willcocks & & & 0.440 & 0.589 & & \\
\hline Saunders & & & & 0.576 & & \\
\hline Rivard & 0.403 & 0.530 & & 0.545 & & \\
\hline Lacity & & & & 0.538 & & \\
\hline Zmud & & 0.481 & & 0.506 & & \\
\hline Benbasat & & 0.475 & & 0.441 & & \\
\hline Hiltz & & & & & 0.930 & \\
\hline Vogel & & & & & 0.887 & \\
\hline
\end{tabular}




\begin{tabular}{|c|c|c|c|c|c|c|}
\hline $\begin{array}{l}\text { 2001-2005 Authors } \\
\text { ( } \geq 4 \text { mean co- } \\
\text { citations) }\end{array}$ & $\begin{array}{l}\text { IS Development \& } \\
\text { Use Thematic } \\
\text { Miscellany }\end{array}$ & $\begin{array}{c}\text { Internet } \\
\text { Application } \\
\text { s Thematic } \\
\text { Miscellany }\end{array}$ & $\begin{array}{l}\text { Qualitative } \\
\text { Methods } \\
\text { Thematic } \\
\text { Miscellany }\end{array}$ & IS Strategy & $\begin{array}{l}\text { Group Work } \\
\text { \& Decision } \\
\text { Support }\end{array}$ & $\begin{array}{c}\text { Inter- } \\
\text { Business } \\
\text { Systems }\end{array}$ \\
\hline Valacich & & & & & 0.885 & \\
\hline Dennis & & & & & 0.861 & \\
\hline Nunamaker & & & & & 0.853 & \\
\hline DeSanctis & & & & & 0.841 & \\
\hline Dickson & & & & & 0.826 & \\
\hline Bostrom & & & & & 0.723 & \\
\hline Wei & & & & & 0.719 & \\
\hline Huber & & & & 0.428 & 0.701 & \\
\hline Alavi & & & & & 0.584 & \\
\hline WatsonRT & & & & & 0.582 & \\
\hline Leidner & & & & & 0.568 & \\
\hline Jarvenpaa & & 0.459 & & & 0.541 & \\
\hline Kriebel & & & & & & 0.851 \\
\hline Kauffman & & & & & & 0.839 \\
\hline Barua & & & & & & 0.808 \\
\hline Mukhopadhyay & & & & & & 0.801 \\
\hline Whinston & & & & & & 0.674 \\
\hline Konsynski & & 0.443 & & 0.460 & & 0.658 \\
\hline Zwass & & & & & 0.413 & 0.488 \\
\hline Kraemer & & & & 0.472 & & 0.488 \\
\hline$\%$ Variance & 9.4 & 16.5 & 13.6 & 16.9 & 12.6 & 7.4 \\
\hline
\end{tabular}

\section{Appendix C}

\section{Derivation of Factor Names from Research Themes}

Factor names were derived by coding the sets of articles co-citing authors within each factor and identifying predominant research themes. We based our initial coding on the classification scheme developed by Swanson and Ramiller (1993) and shown in Table C1. We began our coding by having each author code a sub-set of the co-citing articles in the 1986-1990 period independently. On discussing our codings for this sub-set, we noted that some of the distinctions made by Swanson and Ramiller were difficult to apply consistently. Consequently, as recommended by Miles and Huberman (1994), we developed and redefined the categories to better fit our data, adding more detailed descriptions of each category and heuristics to guide our application of the codes. For articles where none of the existing categories seemed to fit, we used a close reading of the title and abstract to identify newly emergent research themes, which were discussed and agreed by all three authors before being implemented in subsequent coding (Ryan and Bernard 2000). For example, Swanson and Ramiller had no category for the group decision support systems theme, and it was apparent from our initial coding tests of the very first period that this was a distinctive and important category. Other codes, for example knowledge management and internet \& internet users, emerged later in the second and third periods, respectively. When these new codes were added, we reviewed our coding in the previous periods. In each case, we agreed on only a small number of changes to the earlier coding. Within each major category, we developed sub-codes to enable us to apply a fine-grained level of coding initially. While we aggregated these low-level codes to their higher-level categories for subsequent analysis, the detailed sub-codes were invaluable in assisting further post hoc analyses. Our final coding scheme, shown in Table C1, comprised 14 themes, in contrast to the 9 themes used in the Swanson and Ramiller framework. 


\begin{tabular}{|c|c|c|}
\hline $\begin{array}{l}\text { Research Theme } \\
\text { Used in the Current } \\
\text { Study }\end{array}$ & $\begin{array}{l}\text { Swanson \& } \\
\text { Ramiller's Original } \\
\text { Theme }\end{array}$ & Description \\
\hline $\begin{array}{l}\text { Computer supported } \\
\text { cooperative work } \\
\text { (CSCW) and teams }\end{array}$ & CSCW & $\begin{array}{l}\text { CSCW, supporting work and people, including telecommuting and } \\
\text { teleworking; teams, including team interactions and managing teams } \\
\text { (focus on people) }\end{array}$ \\
\hline $\begin{array}{l}\text { Human-computer } \\
\text { interaction }(\mathrm{HCl}) \text { and } \\
\text { interface design }\end{array}$ & $\begin{array}{l}\text { Information and } \\
\text { interface }\end{array}$ & Human and design aspects of $\mathrm{HCl}$; user interface design \\
\hline $\begin{array}{l}\text { Decision support } \\
\text { systems (DSS) }\end{array}$ & $\begin{array}{l}\text { Decision support } \\
\text { and knowledge- } \\
\text { based systems }\end{array}$ & $\begin{array}{l}\text { Information and managerial decision making (the human side of decision } \\
\text { making); DSS design, models, development, implementation, outcome } \\
\text { and applications; Expert systems design, models, development, } \\
\text { implementation, outcome and applications }\end{array}$ \\
\hline $\begin{array}{l}\text { Group decision support } \\
\text { systems (GDSS) }\end{array}$ & & $\begin{array}{l}\text { GDSS design, models, development, implementation, outcome and } \\
\text { applications, including negotiation systems and collaboration systems } \\
\text { (focus on systems) }\end{array}$ \\
\hline $\begin{array}{l}\text { Information system } \\
\text { development (ISD) }\end{array}$ & Systems projects & $\begin{array}{l}\text { Systems development process, methodologies, analysis, design, tools } \\
\text { and techniques; project management; user involvement in ISD; data } \\
\text { modeling and data design; software maintenance }\end{array}$ \\
\hline $\begin{array}{l}\text { Information system } \\
\text { management, } \\
\text { evaluation, and control }\end{array}$ & $\begin{array}{l}\text { Evaluation and } \\
\text { control }\end{array}$ & $\begin{array}{l}\text { Measures of performance and success of systems (but not } \\
\text { costs/benefits); data management; computer resource allocation; IS } \\
\text { security and control; IS ethics; IS personnel and IS management }\end{array}$ \\
\hline IS users & Users & $\begin{array}{l}\text { IS-user relationships; user perceptions and attitudes; user information } \\
\text { evaluation and satisfaction; end-user computing; user training, user } \\
\text { technology acceptance }\end{array}$ \\
\hline $\begin{array}{l}\text { IS strategy and } \\
\text { business outcomes }\end{array}$ & $\begin{array}{l}\text { Economics and } \\
\text { strategy }\end{array}$ & $\begin{array}{l}\text { IS strategic management and business outcomes related to strategy; } \\
\text { globalization; outsourcing; IS in developing countries; IS and culture }\end{array}$ \\
\hline $\begin{array}{l}\text { Interorganizational } \\
\text { systems and e- } \\
\text { business }\end{array}$ & & $\begin{array}{l}\text { Interorganizational systems; electronic data interchange (EDI); supply } \\
\text { chain management (SCM); e-business application projects }\end{array}$ \\
\hline $\begin{array}{l}\text { IS introduction, } \\
\text { diffusion and impact }\end{array}$ & $\begin{array}{l}\text { Introduction and } \\
\text { impact }\end{array}$ & $\begin{array}{l}\text { IS implementation; information technology diffusion; organizational impact } \\
\text { of IT introduction }\end{array}$ \\
\hline $\begin{array}{l}\text { IS research, theory and } \\
\text { education }\end{array}$ & IS research & IS typologies; IS research; IS theory; IS history; IS learning and education \\
\hline $\begin{array}{l}\text { Knowledge } \\
\text { management }\end{array}$ & & Knowledge acquisition; knowledge management; learning organizations \\
\hline $\begin{array}{l}\text { Internet and internet } \\
\text { users }\end{array}$ & & $\begin{array}{l}\text { General internet and e-commerce theories and models; internet } \\
\text { applications and evaluation; internet users; mobile commerce; computer- } \\
\text { mediated communication; user technology acceptance in internet } \\
\text { applications }\end{array}$ \\
\hline $\begin{array}{l}\text { Foundations and other } \\
\text { disciplines }\end{array}$ & & $\begin{array}{l}\text { Research primarily focused in other disciplines, particularly management } \\
\text { science, operations research, and computer science }\end{array}$ \\
\hline
\end{tabular}

The first, second, and third authors independently coded all factors in the 1986-1990 period and the first and third authors coded two factors in the 2001-2005 period. The third author coded all remaining factors, with the first author cross checking a random 10 percent of the articles. All recoding was checked and agreed to by the first and third authors. Agreement between coders before discussion was 90 percent across all articles coded. 


\section{Research Themes Within Factors}

The research themes of the sets of articles co-citing authors in each factor within each period are shown in Table C2, with the major themes for each factor highlighted. The counts and percentages of themes for each factor were calculated as follows: We made the assumption that the research themes of articles co-citing a greater number of authors in a given factor would be more likely to align with the central theme of the factor than the themes of articles that cited fewer of the factor's authors. Hence in determining the contribution of an article to the overall research theme of the factor, we weighted each article by the number of factor authors the article cited. For example, an article by Doll and Torkzadeh (1989) cites 11 authors from one factor and 3 authors from a second factor in the 1986-1990 period, and was coded to the "IS users" research theme. This article was given a weight of 11 in the count of the "IS users" theme in the first factor for period 1986-1990, but a weight of only 3 in the count of that theme in the second factor. This weighting approach helped to differentiate between the themes, particularly for those factors with a high number of cross-loading authors.

\begin{tabular}{|c|c|c|c|c|c|c|c|}
\hline Research Theme & $\begin{array}{l}\text { IS Develop- } \\
\text { ment \& Use } \\
\text { Thematic } \\
\text { Miscellany }\end{array}$ & $\begin{array}{l}\text { Qualitative } \\
\text { Methods } \\
\text { Thematic } \\
\text { Miscellany }\end{array}$ & $\begin{array}{c}\text { Internet } \\
\text { Applications } \\
\text { Thematic } \\
\text { Miscellany }\end{array}$ & $\begin{array}{c}\text { IS } \\
\text { Strategy }\end{array}$ & GDSS & $\begin{array}{c}\text { Inter- } \\
\text { Business } \\
\text { Systems }\end{array}$ & $\begin{array}{c}\text { Foundations } \\
\text { \& Reference } \\
\text { Disciplines }\end{array}$ \\
\hline \multicolumn{8}{|c|}{$1986-1990$} \\
\hline CSCW and teams & $11(1 \%)$ & & & $4(1 \%)$ & $19(7 \%)$ & & $0(0 \%)$ \\
\hline $\mathrm{HCl}$ and interface design & $30(3 \%)$ & & & $4(1 \%)$ & $17(6 \%)$ & & $2(1 \%)$ \\
\hline DSS & $112(10 \%)$ & & & $61(9 \%)$ & $65(23 \%)$ & & $29(20 \%)$ \\
\hline GDSS & $8(1 \%)$ & & & $6(1 \%)$ & $79(27 \%)$ & & $4(3 \%)$ \\
\hline IS development & $204(20 \%)$ & & & $127(19 \%)$ & $8(3 \%)$ & & $27(19 \%)$ \\
\hline $\begin{array}{l}\text { IS management, evaluation and } \\
\text { control }\end{array}$ & $106(10 \%)$ & & & $99(15 \%)$ & $15(5 \%)$ & & $0(0 \%)$ \\
\hline IS users & $220(21 \%)$ & & & $116(17 \%)$ & $27(9 \%)$ & & $4(3 \%)$ \\
\hline $\begin{array}{l}\text { IS strategy and business } \\
\text { outcomes }\end{array}$ & $93(9 \%)$ & & & $97(15 \%)$ & $10(3 \%)$ & & $11(8 \%)$ \\
\hline $\begin{array}{l}\text { Interorganizational systems and e- } \\
\text { business }\end{array}$ & $17(2 \%)$ & & & $32(4 \%)$ & $4(1 \%)$ & & $0(0 \%)$ \\
\hline $\begin{array}{l}\text { IS introduction, diffusion, and } \\
\text { impact }\end{array}$ & $134(13 \%)$ & & & $64(9 \%)$ & $20(7 \%)$ & & $2(1 \%)$ \\
\hline IS research, theory, and education & $84(8 \%)$ & & & $56(8 \%)$ & $15(5 \%)$ & & $25(18 \%)$ \\
\hline Knowledge management & $2(0 \%)$ & & & $2(0 \%)$ & $5(2 \%)$ & & $0 \%$ \\
\hline Internet and Internet users & $0(0 \%)$ & & & $0(0 \%)$ & $0(0 \%)$ & & $0(0 \%)$ \\
\hline Foundations and other disciplines & $16(2 \%)$ & & & $7(1 \%)$ & $6(2 \%)$ & & $38(27 \%)$ \\
\hline \multicolumn{8}{|c|}{ 1991-1995 } \\
\hline CSCW and teams & $19(1 \%)$ & $22(2 \%)$ & & $4(0 \%)$ & $127(11 \%)$ & & $2(1 \%)$ \\
\hline $\mathrm{HCl}$ and interface design & $80(3 \%)$ & $11(1 \%)$ & & $3(0 \%)$ & $56(5 \%)$ & & $2(1 \%)$ \\
\hline DSS & $436(15 \%)$ & $67(7 \%)$ & & $94(8 \%)$ & $221(20 \%)$ & & $77(27 \%)$ \\
\hline GDSS & $43(2 \%)$ & $34(3 \%)$ & & $15(1 \%)$ & $351(30 \%)$ & & $12(4 \%)$ \\
\hline IS development & $445(16 \%)$ & $234(24 \%)$ & & $101(9 \%)$ & $58(5 \%)$ & & $41(14 \%)$ \\
\hline $\begin{array}{l}\text { IS management, evaluation, and } \\
\text { control }\end{array}$ & $303(11 \%)$ & $82(8 \%)$ & & $122(10 \%)$ & $49(4 \%)$ & & $8(3 \%)$ \\
\hline IS users & $398(14 \%)$ & $133(14 \%)$ & & $90(8 \%)$ & $37(3 \%)$ & & $7(2 \%)$ \\
\hline $\begin{array}{l}\text { IS strategy and business } \\
\text { outcomes }\end{array}$ & $340(12 \%)$ & $52(5 \%)$ & & $334(29 \%)$ & $59(4 \%)$ & & $13(4 \%)$ \\
\hline $\begin{array}{l}\text { Interorganizational systems and e- } \\
\text { business }\end{array}$ & $76(2 \%)$ & $9(1 \%)$ & & $113(9 \%)$ & $14(2 \%)$ & & $2(1 \%)$ \\
\hline $\begin{array}{l}\text { IS introduction, diffusion, and } \\
\text { impact }\end{array}$ & $304(11 \%)$ & $187(19 \%)$ & & $148(13 \%)$ & $68(6 \%)$ & & $8(3 \%)$ \\
\hline
\end{tabular}


Table C2. Number and Percentage of Weighted Co-Citing Articles Coded to Each Research Theme in Each Factor for Each Period (Continued)

\begin{tabular}{|c|c|c|c|c|c|c|c|}
\hline Research Theme & $\begin{array}{c}\text { IS Develop- } \\
\text { ment \& Use } \\
\text { Thematic } \\
\text { Miscellany }\end{array}$ & $\begin{array}{l}\text { Qualitative } \\
\text { Methods } \\
\text { Thematic } \\
\text { Miscellany }\end{array}$ & $\begin{array}{c}\text { Internet } \\
\text { Applications } \\
\text { Thematic } \\
\text { Miscellany }\end{array}$ & $\begin{array}{c}\text { IS } \\
\text { Strategy }\end{array}$ & GDSS & $\begin{array}{l}\text { Inter- } \\
\text { Business } \\
\text { Systems }\end{array}$ & $\begin{array}{l}\text { Foundations } \\
\text { \& Reference } \\
\text { Disciplines }\end{array}$ \\
\hline IS research, theory, and education & $212(7 \%)$ & $59(6 \%)$ & & $62(5 \%)$ & $75(6 \%)$ & & $38(13 \%)$ \\
\hline Knowledge management & $14(1 \%)$ & $4(0 \%)$ & & $6(1 \%)$ & $20(2 \%)$ & & $4(1 \%)$ \\
\hline Internet and Internet users & $0(0 \%)$ & $0(0 \%)$ & & $0(0 \%)$ & $0(0 \%)$ & & $0(0 \%)$ \\
\hline Foundations \& other disciplines & $170(6 \%)$ & $94(10 \%)$ & & $81(7 \%)$ & $18(2 \%)$ & & $73(26 \%)$ \\
\hline \multicolumn{8}{|c|}{$1996-2000$} \\
\hline CSCW and teams & $97(3 \%)$ & $45(3 \%)$ & & $19(1 \%)$ & $219(12 \%)$ & $5(2 \%)$ & \\
\hline $\mathrm{HCl}$ and interface design & $29(1 \%)$ & $6(0 \%)$ & & $7(0 \%)$ & $111(6 \%)$ & $0(0 \%)$ & \\
\hline DSS & $295(9 \%)$ & $47(3 \%)$ & & $87(5 \%)$ & $238(13 \%)$ & $29(10 \%)$ & \\
\hline GDSS & $48(1 \%)$ & $16(1 \%)$ & & $16(1 \%)$ & $525(29 \%)$ & $4(2 \%)$ & \\
\hline IS development & $446(13 \%)$ & $228(16 \%)$ & & $140(7 \%)$ & $97(5 \%)$ & $10(4 \%)$ & \\
\hline $\begin{array}{l}\text { IS management, evaluation, and } \\
\text { control }\end{array}$ & $356(11 \%)$ & $158(11 \%)$ & & $192(10 \%)$ & $84(4 \%)$ & $9(3 \%)$ & \\
\hline IS users & $544(16 \%)$ & $66(4 \%)$ & & $135(7 \%)$ & $117(6 \%)$ & $3(1 \%)$ & \\
\hline $\begin{array}{l}\text { IS strategy and business } \\
\text { outcomes }\end{array}$ & $419(12 \%)$ & $220(15 \%)$ & & $535(29 \%)$ & $65(3 \%)$ & $20(7 \%)$ & \\
\hline $\begin{array}{l}\text { Interorganizational systems and e- } \\
\text { business }\end{array}$ & $191(6 \%)$ & $130(9 \%)$ & & $269(14 \%)$ & $38(2 \%)$ & $\begin{array}{c}126 \\
(45 \%)\end{array}$ & \\
\hline $\begin{array}{l}\text { IS introduction, diffusion, and } \\
\text { impact }\end{array}$ & $641(19 \%)$ & $302(21 \%)$ & & $382(20 \%)$ & $121(7 \%)$ & $51(19 \%)$ & \\
\hline IS research, theory, and education & $169(5 \%)$ & $115(8 \%)$ & & $76(4 \%)$ & $105(6 \%)$ & $10(4 \%)$ & \\
\hline Knowledge management & $42(1 \%)$ & $19(1 \%)$ & & $23(1 \%)$ & $22(1 \%)$ & $0(0 \%)$ & \\
\hline Internet and Internet users & $95(3 \%)$ & $89(6 \%)$ & & $13(1 \%)$ & $81(5 \%)$ & $2(1 \%)$ & \\
\hline Foundations and other disciplines & $18(1 \%)$ & $24(2 \%)$ & & $2(0 \%)$ & $13(1 \%)$ & $4(2 \%)$ & \\
\hline \multicolumn{8}{|c|}{ 2001-2005 } \\
\hline CSCW and teams & $20(2 \%)$ & $58(4 \%)$ & $111(4 \%)$ & $55(2 \%)$ & $195(15 \%)$ & $2(0 \%)$ & \\
\hline $\mathrm{HCl}$ and interface design & $4(0 \%)$ & $5(0 \%)$ & $26(1 \%)$ & $2(0 \%)$ & $15(1 \%)$ & $0(0 \%)$ & \\
\hline DSS & $59(6 \%)$ & $36(2 \%)$ & $130(4 \%)$ & $86(3 \%)$ & $54(4 \%)$ & $12(2 \%)$ & \\
\hline GDSS & $6(1 \%)$ & $27(2 \%)$ & $54(2 \%)$ & $55(2 \%)$ & $267(21 \%)$ & $13(2 \%)$ & \\
\hline IS development & $121(13 \%)$ & $241(16 \%)$ & $241(8 \%)$ & $158(6 \%)$ & $111(9 \%)$ & $4(1 \%)$ & \\
\hline $\begin{array}{l}\text { IS management, evaluation, and } \\
\text { control }\end{array}$ & $128(14 \%)$ & $103(7 \%)$ & $207(7 \%)$ & $184(7 \%)$ & $23(2 \%)$ & $6(1 \%)$ & \\
\hline IS users & $142(15 \%)$ & $67(5 \%)$ & $456(15 \%)$ & $133(5 \%)$ & $68(5 \%)$ & $2(0 \%)$ & \\
\hline $\begin{array}{l}\text { IS strategy and business } \\
\text { outcomes }\end{array}$ & $158(16 \%)$ & $215(14 \%)$ & $378(12 \%)$ & $778(30 \%)$ & $62(5 \%)$ & $40(8 \%)$ & \\
\hline Interorganizational systems & $48(5 \%)$ & $96(7 \%)$ & $385(13 \%)$ & $491(18 \%)$ & $57(4 \%)$ & $\begin{array}{c}301 \\
(55 \%)\end{array}$ & \\
\hline $\begin{array}{l}\text { IS introduction, diffusion, and } \\
\text { impact }\end{array}$ & $126(14 \%)$ & $272(19 \%)$ & $319(11 \%)$ & $331(12 \%)$ & $56(4 \%)$ & $44(8 \%)$ & \\
\hline IS research, theory, and education & $42(5 \%)$ & $195(13 \%)$ & $137(5 \%)$ & $101(4 \%)$ & $69(5 \%)$ & $11(2 \%)$ & \\
\hline Knowledge management & $23(2 \%)$ & $69(5 \%)$ & $111(4 \%)$ & $115(4 \%)$ & $211(16 \%)$ & $18(3 \%)$ & \\
\hline Internet and Internet users & $54(6 \%)$ & $57(4 \%)$ & $429(14 \%)$ & $163(6 \%)$ & $109(9 \%)$ & $85(15 \%)$ & \\
\hline Foundations and other disciplines & $12(1 \%)$ & $21(2 \%)$ & $13(0 \%)$ & $16(1 \%)$ & $7(1 \%)$ & $7(1 \%)$ & \\
\hline
\end{tabular}




\section{Analyses of Research Themes of Sets of Co-Citing Articles}

The predominant themes - that is, generally those two or three themes that accounted for at least 50 percent of the co-citing articles within a factor - were used to make an initial determination of the primary focus of the factor. All three authors reviewed the research themes for each factor and discussed and agreed on names for the factors that we believed best captured the essence of the major themes in the factor. As the characteristics of each factor began to crystallize, we considered the research areas of the leading foundational authors in each factor, recognizing that, over time, many of these foundational authors have worked, and have been cited, in multiple areas. We also reviewed the editorial policies of the journals publishing the co-citing articles to ensure that the naming made sense. (Details about the journals publishing co-citing articles in each factor are presented in Appendix D.)

This approach worked well for the IS Strategy, Group Work \& Decision Support (GDSS), Foundations \& Reference Disciplines, and InterBusiness Systems factors, but the remaining three factors all included a similar distribution of research themes and required further analysis of methodological and contextual characteristics of the co-citing articles to establish the nature of the common influence of the foundational authors.

The factor finally called IS Development \& Use Thematic Miscellany was the most difficult to name. For the first three periods it accounted for the most variance and clearly held a central position in the field, but its set of research themes were relatively diverse. Thus we reviewed the co-citing articles and the author membership of the factor closely, before concluding that this factor has formed around key authority figures in the field and encompasses a broad set of system- and relationship-oriented research into information systems- their development, their implementation, their impact, and their use by individuals and within organizations.

While the research themes of the Internet Applications Thematic Miscellany factor also included similar development, implementation, impact, and use issues, there was a clear emphasis in the set of co-citing articles on the Internet as the context of inquiry. The Qualitative Methods Thematic Miscellany factor, however, was more difficult, with its major theme, related to research methodology, confirmed only after a consideration of the journal outlets and the author membership and factor time lines (discussed in Appendices D and E, respectively).

\section{Appendix D}

\section{Journal Outlets for Each Factor and Across the Field}

The top 10 journals publishing articles citing authors in each factor during the most recent 10 years of the factor's coverage are shown in Table D1. For all factors except Foundations \& Reference Disciplines, we focused on the most recent 10 years (1996-2005), because the Journal of Management Information Systems, one of the more important IS journals (Saunders 2006), is only indexed by Web of Science from 1996 onward and hence is under-represented in counts of journals in the earlier two time periods. Since the Foundations \& Reference Disciplines factor only appears in the first two time periods, we report the publishing journals from 1986-1995 for this factor. For all factors except the Foundations \& Reference Disciplines factor, Information \& Management, MIS Quarterly, and Journal of Management Information Systems dominate the rankings, but differences in the other journals appearing in the top 10 for each factor help to distinguish between factors. For example, Information Systems Journal, ranked the third highest publishing outlet for the Qualitative Methods Thematic Miscellany factor, gives strong support to qualitative research in its editorial policies, helping to confirm our choice of name for this factor. Similarly, the journal outlets for the GDSS factor include Decision Support Systems in third place, while the Foundations \& Reference Disciplines factor shows a heavy emphasis on operations research journals.

We also combined all of the co-citing bibliographies and removed duplicates (since articles co-citing cross-loading authors were included in the bibliographies for both cross-loaded factors) to gain a picture of the overall publication patterns for the field. A total of 3,818 unique articles were published across 179 different journals. Sixty of these journals each published at least 10 articles, while the top 20 journals, shown in Table D2, contained 72 percent of the articles. Not surprisingly, seven of the eight journals in the AIS senior scholars' expanded basket of journals (MIS Quarterly, Information Systems Research, Journal of Management Information Systems, Information Systems Journal, European Journal of Information Systems, Journal of Information Technology, and Journal of Strategic Information Systems) feature in the top 20 journals in Table D2, but the leading ranking of Information \& Management both overall, and in the individual factor rankings, may be more surprising. 
Table D1. Top Ten Journals Publishing Articles Citing Authors in Each Factor (for Periods 1996-2005 for All Factors Except Foundations; Foundations for Periods 1986-1995)

\begin{tabular}{|c|c|c|c|c|c|c|c|}
\hline Rank & IS Strategy & $\begin{array}{c}\text { IS } \\
\text { Development \& } \\
\text { Use Thematic } \\
\text { Miscellany } \\
\end{array}$ & $\begin{array}{c}\text { Internet } \\
\text { Applications } \\
\text { Thematic } \\
\text { Miscellany }\end{array}$ & $\begin{array}{c}\text { Qualitative } \\
\text { Methods Thematic } \\
\text { Miscellany }\end{array}$ & GDSS & $\begin{array}{c}\text { Inter- } \\
\text { Business } \\
\text { Systems }\end{array}$ & $\begin{array}{c}\text { Foundations and } \\
\text { Reference } \\
\text { Disciplines } \\
(1986-1995)\end{array}$ \\
\hline 1 & I\&M & I\&M & I\&M & I\&M & I\&M & JMIS & JORS \\
\hline \multirow{2}{*}{2} & MISQ & MISQ & JMIS & MISQ & MISQ & I\&M & MISQ \\
\hline & & & & & & ISR & Systems Research \\
\hline \multirow{2}{*}{3} & JIT & EJIS & $\mathrm{JCIS}$ & ISJ & DSS & & \\
\hline & & & MISQ & & & & \\
\hline 4 & EJIS & $\mathrm{JCIS}$ & & JIT & JMIS & DSS & Omega \\
\hline 5 & JMIS & JIT & ISR & EJIS & ISR & MISQ & EJOR \\
\hline \multirow{2}{*}{6} & IJIM & IJIM & EJIS & JSIS & $\mathrm{JCIS}$ & IJEC & Interfaces \\
\hline & JSIS & ISR & JSIS & & & & \\
\hline \multirow{2}{*}{7} & & & & JMIS & IJHCS & JIT & I\&M \\
\hline & & & & & & MS & \\
\hline 8 & ISR & JSIS & ISJ & IJIM & EJOR & & DSS \\
\hline 9 & $\mathrm{JCIS}$ & ISJ & IJIM & ISR & EJIS & JOCEC & IEEE Transactions \\
\hline 10 & ISJ & JMIS & DSS & Information Society & MS & $\mathrm{JCIS}$ & \\
\hline
\end{tabular}

†The journal Systems Research was a publishing outlet for some researchers co-citing the authors in the "Foundations" factor. The journal merged with Behavioral Science in 1997 and continues as Systems Research \& Behavioral Science.

Notes: DSS = Decision Support Systems; EJIS = European Journal of Information Systems; EJOR = European Journal of Operational Research; IEEE Transactions = IEEE Transactions on Systems, Man and Cybernetics Management Science; IJEC = International Journal of Electronic Commerce; IJHCS = International Journal of Human-Computer Studies; IJIM = International Journal of Information Management; I\&M = Information \& Management; ISJ = Information Systems Journal; ISR = Information Systems Research; JCIS = Journal of Computer Information Systems; JIT = Journal of Information Technology; JMIS = Journal of Management Information Systems; JORS = Journal of the Operational Research Society; JOCEC = Journal of Organizational Computing and Electronic Commerce; JSIS = Journal of Strategic Information Systems; MISQ = MIS Quarterly; MS = Management Science; Omega = Omega-International Journal of Management Science 


\begin{tabular}{|c|c|c|}
\hline Journal & $\begin{array}{c}\text { Number of } \\
\text { Articles } \\
\text { Out of } 3,818 \text { Total }\end{array}$ & Percent \\
\hline Information \& Management & 444 & $12 \%$ \\
\hline MIS Quarterly & 348 & $9 \%$ \\
\hline Journal of Computer Information Systems & 167 & $4 \%$ \\
\hline International Journal of Information Management & 161 & $4 \%$ \\
\hline Journal of Information Technology & 155 & $4 \%$ \\
\hline Information Systems Research & 155 & $4 \%$ \\
\hline Decision Support Systems & 146 & $4 \%$ \\
\hline European Journal of Information Systems & 133 & $3 \%$ \\
\hline Journal of Management Information Systems & 133 & $3 \%$ \\
\hline Omega-International Journal of Management Science & 132 & $3 \%$ \\
\hline Information Systems Journal & 124 & $3 \%$ \\
\hline Journal of Strategic Information Systems & 109 & $3 \%$ \\
\hline Behaviour \& Information Technology & 97 & $3 \%$ \\
\hline Management Science & 84 & $2 \%$ \\
\hline European Journal of Operational Research & 79 & $2 \%$ \\
\hline Communications of the ACM & 64 & $2 \%$ \\
\hline International Journal of Human-Computer Studies & 64 & $2 \%$ \\
\hline Journal of the Operational Research Society & 54 & $1 \%$ \\
\hline Industrial Management \& Data Systems & 49 & $1 \%$ \\
\hline International Journal of Electronic Commerce & 40 & $1 \%$ \\
\hline
\end{tabular}

\section{Appendix E}

\section{Key Foundational Authors Associated with Factors over Time}

To help confirm our factor interpretations, we created timelines of the author composition of each factor. We traced the movement of high loading authors (those loading at least 0.8 on at least one factor in one of the four periods), starting from Culnan's (1987) factors, and tracked how factor membership changed through the following periods. Figure E1 shows the detailed movement of high loading authors, while a summary timeline showing how each factor has evolved through the periods is shown in Figure 8 in the body of the article. As shown in Figure E1, authors from four of Culnan's five factors split and recombine in subsequent periods. Culnan's fifth factor, MIS curriculum, which comprised only three authors, has no corresponding factor in any subsequent period.

The time lines revealed that the authors in the 1986-1990 IS Development \& Use Thematic Miscellany factor split into two factors in subsequent periods, and yet the research themes for both factors seemed very similar. A consideration of the author membership of the "breakaway" factor led us to speculate that these authors were being cited by researchers with an interest in the use of qualitative methods to examine key research topics in the IS field. In order to further confirm our understanding, we undertook a secondary coding of the articles co-citing authors in the parent IS Development \& Use Thematic Miscellany factor and the offspring factor, categorizing articles according to whether they reported quantitative or qualitative methods. We also examined the journal outlets for the offspring factor, as discussed previously in Appendix D, before agreeing on the final name of Qualitative Methods Thematic Miscellany. 


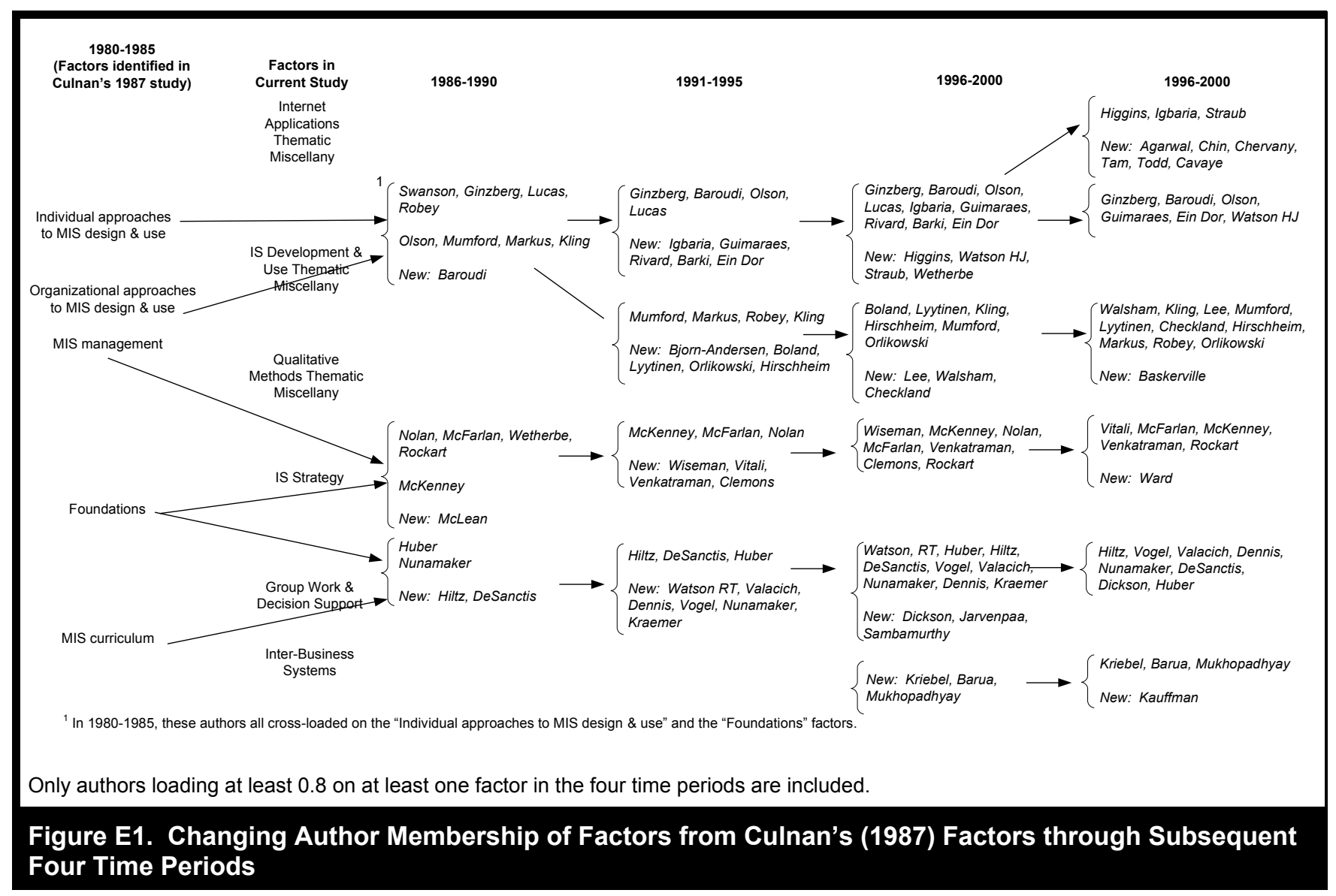

\section{Appendix F}

\section{Technical Limitations}

Although this study avoids the problems associated with the limited selection of journals for analysis seen in earlier citation studies (Grover et al. 2006; Wade et al. 2006), there are other limitations arising from the author co-citation research approach used. In particular, the final choice of influential authors and the analysis of researcher perspectives of these authors' key research areas through co-citation counts rely on an initial citation analysis. In this respect it is interesting to note differences between our author set and the one developed by Lowry et al. (2007), and to note once again the impact of basket of journal choices. Lowry et al. limited their study to authors published in MIS Quarterly, Information Systems Research, and the IS articles from Management Science and focused solely on citations, not on publication counts. In our study, we cast a much broader net across the wide range of constituencies that comprise the IS field, in order to identify a body of contributors whose whole corpus of work has been influential over time, either through a high number of publications in premier IS journals, or by outstanding contributions through books or service to the field. Thus, we drew our publication counts from a wider set of journals (MIS Quarterly, Information Systems Research, Journal of Management Information Systems, European Journal of Information Systems, and Information Systems Journal) and established a high bar of number of publications for inclusion in the author set. As a result, only 45 of the authors reported on in Lowry et al. are included among our final authors drawn from publication counts. In addition, our set includes 45 authors selected on a reputational basis, of which only 4 appear in Lowry's list. However, these reputational authors have had significant impact on the field, as evidenced by citations to their work.

We also note differences in the citation counts for authors appearing on both the Lowry et al. list and our list, and that the total number of citations to our key authors is lower than might be expected from other recent studies such as Grover et al. (2006) and Wade et al. (2006). 
These differences result from our decision to limit citing references to information systems categories, in order to focus on author influence within the IS field, rather than beyond it. The Grover et al. and Wade et al. studies were aimed at examining the IS field's position within a larger body of disciplines to determine the extent to which IS makes a contribution beyond the IS field, while Lowry et al. used citation analysis to determine the impact of IS institutions, articles, and authors, both within and beyond the IS field. In contrast, our goal was to identify those authors who have had seminal impact within the IS field and hence might be expected to have provided leadership on the direction of the field. A number of our leading authors are highly published in non-IS journals and if we had included all citations to their work we would get a very different picture of the most influential authors-however, they would no longer necessarily be the most influential within the field.

An examination of the lower ranked authors in Table A1 in Appendix A reveals that 21 have no publications available to cite in the first 5-year period. The impact of these researchers on the IS field may well be seen more substantially in future years. Some of the other low-cited authors are surprising, however, and highlight other limitations of using co-citation analysis as a tool for examining influence in a field. For example, Langefors has been recognized with a Leo Award for his contributions to IS research and practice, particularly in Scandinavia, and Neumann, an AIS Fellow, has been recognized for his impact on IS teaching, practice, and research in Israel. Both of these authors are under-recognized by our approach and illustrate two biases set by our research design. First, our focus on English-language articles disadvantages researchers whose influence has been primarily in non-English speaking countries. Second, the citation analysis approach under-recognizes those whose contribution has been primarily in teaching and service areas. Another limitation is that second or subsequent authors of books do not get citation credits under the SSCI citation indexing system, and this is reflected in Carlson's low citation count. Carlson was a second author with Sprague on a key book, Building Effective Decision Support Systems, and it is worth noting that 166 of the 311 citations recorded for Carlson's co-author, Sprague, are citations to their co-authored book. Finally, Jackson has been highly cited in the Web of Science Science Citation Index Expanded database and his low ranking here reflects our deliberate exclusion of citations in more technical computer science areas in order to keep our focus as tightly as possible on the IS field.

\section{References}

Bayer, A. E., Smart, J. C., and McLaughlin, G. W. 1990. "Mapping Intellectual Structure of a Scientific Subfield through Author Cocitations," Journal of the American Society for Information Science and Technology (41:6), pp. 444-452.

Chua, C., Cao, L., Cousins, K., and Straub, D. 2002. "Measuring Researcher-Production in Information Systems," Journal of the Association for Information Systems (3:6), pp. 145-215.

Culnan, M. J. 1986. “The Intellectual Development of Management Information Systems 1972-1982: A Co-Citation Analysis,” Management Science (32:2), pp. 156-172.

Culnan, M. J. 1987. "Mapping the Intellectual Structure of MIS, 1980-1985: A Co-Citation Analysis," MIS Quarterly (11:3), pp. 341-353.

Culnan, M. J., O’Reilly III, C. A., and Chatman, J. A. 1990. "Intellectual Structure of Research in Organizational Behavior, 1972-1984: A Cocitation Analysis," Journal of the American Society for Information Science and Technology (41:6), pp. 453-458.

Doll, W. J., and Torkzadeh, G. 1989. “A Discrepancy Model of End-User Computing Involvement,” Management Science (35:10), pp. $1151-1171$

Ellis, D., Allen, D., and Wilson, T. 1999. "Information Science and Information Systems: Conjunct Subjects Disjunct Disciplines," Journal of the American Society for Information Science (50:12), pp. 1095-1107.

Grover, V., Ayyagari, R., Gokhale, R., Lim, J., and Coffey, J. 2006. "A Citation Analysis of the Evolution and State of Information Systems Within a Constellation of Reference Disciplines," Journal of the Association for Information Systems (7:5), pp. $270-325$.

Lowry, P. B., Karuga, G. G., and Richardson, V. J. 2007. “Assessing Leading Institutions, Faculty, and Articles in Premier Information Systems Research Journals," Communications of the Association for Information Systems (20), pp. 142-203.

McCain, K. W. 1990. "Mapping Authors in Intellectual Space: A Technical Overview," Journal of the American Society for Information Science (41:6), pp. 433-443.

Miles, B. M., and Huberman, A. M. 1994. Qualitative Data Analysis: An Expanded Sourcebook (2 ${ }^{\text {nd }}$ ed.), London: Sage.

Ponzi, L.J. 2002. "The Intellectual Structure and Interdisciplinary Depth of Knowledge Management: A Bibliometric Study of its Early Stage of Development," Scientometrics (55:2), pp. 259-272.

Ryan, G. W., and Bernard, H. R. 2000. "Data Management and Analysis Methods," in Handbook of Qualitative Research, N. K. Denzin and Y. A. Lincoln (eds.), Thousand Oaks, CA: Sage, pp. 769-802.

Saunders, C. 2006. "MIS Journal Rankings," available online at www.isworld.org/csaunders/rankings.htm; retrieved July $19,2006$.

Swanson, E. B., and Ramiller, N. C. 1993. "Information Systems Research Thematics: Submissions to a New Journal, 1987-1992," Information Systems Research (4:4), pp. 299-330.

Wade, M., Biehl, M., and Kim, H. 2006. "Information Systems Is Not a Reference Discipline (And What We Can Do about It)," Journal of the Association for Information Systems (7:5), pp. 247-269.

White, H. D., and McCain, K. W. 1998. "Visualizing a Discipline: An Author Co-Citation Analysis of Information Science, 1972-1995," Journal of the American Society for Information Science (49:4), pp. 327-355. 